\title{
Galactic Interactions: New Source of Lithium?
}

\author{
Tijana Prodanović ${ }^{* \dagger}$ \\ University of Novi Sad, Serbia \\ E-mail: prodanvcedf.uns.ac.rs
}

\section{Tamara Bogdanović}

Georgia Institute of Technology, USA

\section{Dejan Urošević $\$$}

University of Belgrade, Serbia

Given the persisting problem of matching the primordial lithium abundance predictions with abundances observed in low-metallicity systems, it is important to account for all possible sources and sink of lithium, especially at early epochs. We investigate the impact of close galactic fly-bys on lithium nucleosynthesis through interactions of cosmic rays, additional to standard galactic cosmic rays, which get accelerated in large-scale tidal shocks that arise in galactic interactions. This is important for low-metallicity systems, especially if such system is a smaller of the two interacting objects. For our test case we pick Small Magellanic Cloud (SMC) which has experienced few close interactions with neighboring galaxies. We show that only a few close galactic fly-bys would be sufficient to significantly (at the level of lithium abundance produced in standard galactic cosmic-ray nucleosynthesis) contaminate the SMC gas with extra lithium made in tidal cosmic-ray interactions. This is especially important in the light of the recent first gas-phase measurement of lithium in the SMC, which serves to test the nature of the primordial lithium problem. Our results indicated that the problem with lithium persists even in the gas-phase lowmetallicity environment such is the SMC, and that its solution should be sought in the form of the new physics.

\section{Nuclei in the Cosmos}

7-11 July, 2014

Debrecen, Hungary

\footnotetext{
*Speaker.

${ }^{\dagger}$ The work of T.P. is supported in part by the Ministry of Education, Science and Technological Development of the Republic of Serbia under project numbers 171002 and 176005.

${ }^{\ddagger}$ Support for T. B. was in part provided by NASA through Einstein Postdoctoral Fellowship Award No. PF9-00061 issued by the Chandra X-ray Observatory Center, which is operated by the Smithsonian Astrophysical Observatory for and on behalf of NASA under Contract No. NAS8-03060.

$\S$ The work of D. U. is supported by the Ministry of Education, Science and Technological Development of the Republic of Serbia under Project No. 176005.
} 


\section{Introduction}

Predicted abundance of primordial ${ }^{7} \mathrm{Li}$ made during the Big Bang nucleosynthesis (BBN) [1] is found to be 3-4 times higher than its pre-galactic abundance measured in low metallicity halo stars [2]. It is still unclear whether this discrepancy comes from some systematics related to lithium measurements in photospheres of halo stars, or is it real and as such is the result of some postBBN destruction of lithium in stars themselves or in pre-galactic gas, or finally, is it due to some new physics which would change the primordial abundances. Measurements of lithium in the interstellar medium of some low metallicity systems [3] would be crucial in testing whether the problem lies within stellar observations and modeling or is in the physics. One such measurement was recently done in the Small Magellanic Cloud (SMC) at the metallicity 20\% of Solar [4]. Besides primordial ${ }^{7} \mathrm{Li}$, both lithium isotopes are also made in cosmic-ray interactions in the in ratio varying between ${ }^{7} \mathrm{Li} /{ }^{6} \mathrm{Li}=1.3-2$ depending on the cosmic-ray spectrum [5]. Li abundance measured in the SMC was found to be consistent with predicted primordial value only if very little lithium was made post-BBN by galactic cosmic rays (GCRs) [4].

Here we point out to potential importance of galactic interactions on lithium abundances. Galactic mergers and close fly-bys give rise to large-scale shocks in the gas of interacting galaxies [6]. Such shocks would accelerate tidal cosmic rays (TCRs) which would not be directly accompanied with increase in metallicity, as is expected from GCRs which originate from supernova remnants, but would make lithium [7]. In Prodanović et al. 2014 [7] we have demonstrated that these shocks have sufficient energy and could result in cosmic-ray fluxes high enough to produce significant amount of Li. This is especially important for low-metallicity sites such is the Small Magellanic Cloud (SMC) which have experienced galaxy harassment in their history [8].

\section{Energy Requirement}

Before proceeding one must make sure that available energy budged is sufficient for a proposed lithium production mechanism to work. Thus, energy released in large scale tidal shocks should account for the energy necessary to produce the level of Li measured in these systems. Available energy from a fly-by event is the kinetic energy of the encounter which is about $E_{\text {kin }} \approx 4 \times 10^{57} \mathrm{erg}$ when the separation between galaxies is $50 \mathrm{Mpc}$ and with the mass of the primary (larger) galaxy is $10^{12} M_{\odot}$ [7]. Simulations show that such encounters are more damaging for the satellite galaxy [9]. Because of shallower potential well gas of the satellite galaxy can be strongly shocked. ${ }^{6} \mathrm{Li}$ is produced in cosmic-ray interactions only, dominantly via $\alpha \alpha \rightarrow^{6} \mathrm{Li}+\ldots$ the fusion channel, which makes is a perfect cosmic-ray dosimeter and places energy requirements on any cosmic-ray source. Energy required for ${ }^{6} \mathrm{Li}$ production is about $16 \mathrm{erg} /$ nucleus [10]. Thus, energy required to pollute total ISM gas of mass $10^{9} M_{\odot}$ of a satellite galaxy at solar-metallicity with lithium is then $E_{6 \mathrm{Li}} \approx 3 \times 10^{57} \mathrm{erg}[7]$.

In the case of the Small Magellanic Cloud with gas mass of $3 \times 10^{8} M_{\odot}$, the energy required to pollute all gas of the SMC with lithium abundance expected at 1/5 solar metallicity of this system would be $E_{6 \mathrm{Li}} \approx 2 \times 10^{56} \mathrm{erg}$. Taking the Milky Way as the primary galaxy, the energy available for particle acceleration and lithium production, which comes from MW-SMC interaction at a present 
day distance of $61 \mathrm{kpc}$, is thus $E_{k i n, S M C-M W} \sim 10^{58}$ erg i.e. only about $2 \%$ of this energy would have to go into particle acceleration to account all lithium [7].

\section{Tidal vs. Galactic Cosmic Rays}

Tidal cosmic rays can be a potentially important source of lithium in some systems if they can produce $\mathrm{Li}$ in comparable amounts to what GCRs have produced over history of that system. On one hand tidal cosmic ray population would arise due to rare fly-by events and would be present in episodes but would be accelerated in a large volume of gas through which the tidal shock moves. On the other hand there are standard galactic cosmic rays which are constantly injected in starforming galaxies but are accelerated in smaller scale regions - in gas traversed by the supernova shock. Since GCRs are constantly accelerated in SNRs while TCRs arise only during isolated flyby events, for TCRs to be as efficient in producing lithium as GCRs, they have to be accelerated in a much larger volume to have sufficiently high fluxes and here we estimate how large that volume needs to be. Though mechanisms are different, the assumption is that the underlying physics of cosmic-ray acceleration for both CR populations is similar.

Fraction of a satellite galaxys gas that must be shocked in order to sufficiently accelerate a large flux of TCRs which would produce as much lithium in one fly-by event, as GCRs have produced over the entire history, is estimated to be $M_{\text {shock }} / M_{\text {gas }} \sim 8$ [7]. This means that the entire solar-metallicity gas of the satellite galaxy needs to be shocked 8 times in order for tidal cosmic rays to produce as much lithium as GCRs have produced over the history of the galaxy. This estimate was done under assumptions that acceleration efficiency is the same for both cosmic-ray populations, that GCRs are efficiently accelerated up to the supernova remnant radius of $10 \mathrm{pc}$, that the interstellar medium number density is $1 \mathrm{~cm}^{-3}$. The main underlying assumption and the main caveat is the assumption of the lifetime of TCR population which was taken to be 1 Gyr. The lifetime of tidal shocks and this cosmic-ray population will be analysed in a followup work in numerical modeling. In the case of the SMC where the metallicity is not solar but 1/5 of solar, this ratio becomes $M_{\text {shock}} / M_{\text {gas }} \sim 2$ i.e. the entire gas of the SMC would need to be shocked only twice to allow for enough TCRs to be accelerated to produce as much lithium as GCRs did over the history [7].

Another consequence of the existence of additional cosmic ray population would be the change in the isotopic abundance ratio of lithium due to the fact that ${ }^{6} \mathrm{Li}$ is only made in cosmic-ray interactions and thus serves as a cosmic-ray dosimeter, while ${ }^{7} \mathrm{Li}$ was also made in theBig Bang nucleosynthesis. At the SMC metallicity, producing the same amount of lithium with both GCRs and additional cosmic-ray population would result in the lithium isotopic ratio of ${ }^{7} \mathrm{Li} /{ }^{6} \mathrm{Li} \sim 10$. This isotopic ratio would be anomalously low compared to ratio ${ }^{7} \mathrm{Li} /{ }^{6} \mathrm{Li} \sim 18$ that is expected at SMC metallicity if all observed lithium was only made by GCRs. However, new lithium observations in the SMC gas indicate isotopic ratio of ${ }^{7} \mathrm{Li} /{ }^{6} \mathrm{Li} \sim 10[4]$, consistent with our result [7].

\section{Conclusion}

Close fly-bys between galaxies can result in large scale tidal shocks in the galactic gas which 
would accelerate tidal cosmic rays. These shocks would arise in a minor satellite galaxy, such is the Small Magellanic Cloud, which has already suffered galaxy harassment by the Milky Way and Large Magellanic Cloud. Tidal shocks would give rise to additional cosmic-ray population (other than standard GCRs accelerated in SNRs) which would lead to extra production of both lithium isotopes. We estimate that it would be sufficient for gas of the SMC to be tidally shocked only about twice in order for TCRs to produce as much $\mathrm{Li}$ as GCRs have produced over history. Additional Li produced by TCRs would result in anomalously low ${ }^{7} \mathrm{Li} /{ }^{6} \mathrm{Li}$ ratio in SMC, consistent with most recent observations of lithium in the gas phase of the SMC [4]. This implies that significant amount of SMC Li was produced post-BBN and must be corrected for when comparing to predictions of primordial Li abundance. As a result, SMC Li gas phase abundance is also below primordial Li abundance further indicating the need for new physics in order to solve the lithium problem. The presence of any additional cosmic-ray population (structure-formation cosmic rays, tidal cosmic rays etc.) will also have other important consequences. Cosmic-ray presence also inevitably leads to radio emission which can be used to test for the presence of TCRs. Since tidal interactions would also trigger and result in enhanced star-formation activity which would result in enhanced GCR flux, to be able to distinguish between the two cosmic-ray population one would need to look for systems that are currently interacting where there is no indication of enhanced star-formation rate in the smaller of the two interacting galaxies. Detecting an enhanced radio emission in such system (in the smaller one) would be consistent with having another cosmic-ray population, such are TCRs, present. Similar would be expected of gamma-ray emission. Finally, we note that the presence of additional cosmic-ray population would also have important consequences for the FIR-radio correlation [12] which is important for determining galactic star-formation rates. The presence of TCRs would result in enhanced radio emission which would not be accompanied by corresponding enhancement of FIR emission which is expected if FIR-radio correlation is assumed to hold. This would lead to overestimate of star-formation rates of such systems, therefore it is of great importance for many reasons to test for the presence of tidal cosmic rays.

\section{References}

[1] R. H. Cyburt, B. D. Fields, and K. A. Olive, JCAP, 11, 12 (2008)

[2] F. Spite and M. Spite, Astron. and Astrophys., 115, 357 (1982)

[3] T. Prodanović and B. D. Fields, Astrophys. J. Lett., 616, L115 (2004)

[4] J. C. Howk, N. Lehner, B. D Fields and G. J. Mathews, Nature, 489, 121 (2012)

[5] B. D. Fields and T. Prodanović, Astrophys. J. 623, 877 (2005)

[6] B. Moore, N. Katz, G. Lake, A. Dressler and A. Oemler, Nature, 379, 613 (1996)

[7] T. Prodanović, T. Bogdanović and D. Urošević, Phys. Rev. D 87, 103014 (2013)

[8] J. Diaz, and K. Bekki, MNRAS, 413, 2015 (2011)

[9] S. Callegari, S. Kazantzidis, L. Mayer, et al., Astrophys. J. 729, 85 (2011)

[10] N. Prantzos, Astron. and Astrophys., 448, 665 (2006)

[11] A. Dobardžić and T. Prodanović, Astrophys. J. 782, 109 (2014)

[12] T. de Jong, U. Klein, R. Wielebinski, and E. Wunderlich, Astron. and Astrophys., 147, L6 (1985) 